\title{
Prevalencia de complicaciones mayores en nefrolitotomía percutánea en una clínica de tercer nivel en Medellín entre 2015-2017
}

\section{Prevalence of Major Complications in Percutaneous Nephrolithotomy in a Third Level Clinic in Medellín Between 2015-2017}

\author{
Rodrigo Toro-Bermúdez ${ }^{10}$ David Ruiz-Londoño ${ }^{2}$ Johana Andrea Benavides-Martínez ${ }^{1}$ \\ Diego Alberto Velásquez-Ossa ${ }^{2}$ Rafael Ignacio Castellanos ${ }^{2}$ Matilde Henao-Velásquez ${ }^{1}$ \\ Andrés Gómez-Hoyos ${ }^{1}$ Juan Camilo Londoño-Cano ${ }^{3}$ \\ ${ }^{1}$ Residente de urología, Universidad CES, Antioquia, Medellín, Colombia
${ }^{2}$ Urólogo, Clínica CES, Antioquia, Medellín, Colombia
${ }^{3}$ Médico general, Universidad CES, Antioquia, Medellín, Colombia \\ Address for correspondence Rodrigo Toro-Bermúdez, MD, Residente \\ de urología, Universidad CES, Antioquia, Medellín, Antioquia. Calle \\ 10a \#22-04, Colombia (e-mail: rodrigotorobe@hotmail.com).
}

Urol Colomb 2020;29:77-83.

\section{Resumen \\ Palabras clave \\ - nefrostomía \\ - percutánea \\ - nefrolitiasis \\ - complicaciones \\ - postoperatorias \\ - derivación urinaria \\ - prevalencia}

Objetivo La Urolitiasis constituye un motivo de consulta común, con una alta incidencia y prevalencia. Cuando los cálculos renales son mayores a $20 \mathrm{~mm}$, la primera línea de tratamiento es la nefrolitotomía percutánea. En Colombia existe poca información sobre la evaluación de las características de los pacientes y las complicaciones asociadas a ese procedimiento quirúrgico. Este estudio, busca determinar la prevalencia de las complicaciones en nefrolitotomía percutánea en una clínica de Medellín entre los años 2015 y 2017.

Métodos Estudio observacional retrospectivo en el que se analiza la prevalencia de complicaciones mayores que presentaron los pacientes sometidos a nefrolitotomía percutánea en una clínica de tercer nivel en la ciudad de Medellín entre los años 2015 y 2017.

Resultados a 176 pacientes se les realizó Nefrolitotomía percutánea entre el 2015 y el 2017. Se encontró una prevalencia de complicaciones mayores de $11,4 \%$. El 60,2\% de los pacientes que presentaron complicaciones fueron mujeres y la edad media fue de 45,1 años ( $D E \pm 12,5$ años). El $60 \%$ de los cálculos eran coraliformes. El $45 \%$ ubicados en el caliz inferior; siendo bilaterales en el $40 \%$ de ellos. La complicación más frecuente, fueron los cálculos residuales en el 6,8\% seguido de complicaciones pulmonares en el $6,3 \%$ e infecciosas en el $6,3 \%$. received October 19, 2018

accepted April 2, 2019
DOI https://doi.org/ 10.1055/s-0039-1688705. ISSN 0120-789X. e ISSN 2027-0119.
Copyright $\odot$ 2020, Sociedad Colombiana License terms de Urología. Publicado por Thieme Revinter Publicações Ltda., Rio de Janeiro, Brazil. Todos los derechos reservados.

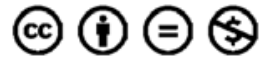




\begin{abstract}
Keywords

- percutaneous nephrolithotomy

- nephrostomy

- stone

- prevalence

- complications

Objective Urolithiasis is a common reason to consult in medical practice. When stones are greater than $20 \mathrm{~mm}$, the first line of treatment is percutaneous nephrolithotomy. This study aims to determine the prevalence of complications in percutaneous nephrolithotomy in a clinic in Medellín - Colombia between 2015 and 2017.

Methods We present a retrospective observational study in which the prevalence of major complications in patients undergoing percutaneous nephrolithotomy was analyzed in a third level clinic in the city of Medellin - Colombia between 2015 and 2017.

Results 176 patients underwent percutaneous nephrolithotomy between 2015 and 2017. A prevalence of complications greater than $11.4 \%$ was found. $60.2 \%$ of the patients who presented complications were women and the average age was 45.1 years ( $S D \pm 12.5$ years). $60 \%$ of the stones were staghorn. $45 \%$ located in the lower calyx; being bilateral in $40 \%$ of cases. The most frequent complication was residual stones in $6.8 \%$ followed by pulmonary complications in $6.3 \%$ and infectious in $6.3 \%$. Conclusions Percutaneous nephrolithotomy represents a relatively safe surgical procedure for the management of patients with a high lithiasic load with a relatively low percentage of complications.
\end{abstract}

Conclusiones La nefrolitotomía percutánea representa un procedimiento quirúrgico relativamente seguro para el abordaje de pacientes con alta carga litiásica con un porcentaje de complicaciones relativamente bajo.

\section{Introducción}

La urolitiasis, definida como una masa sólida compuesta de pequeños cristales y localizada en el aparato urinario, es un problema de salud pública poco estudiado. Poco se sabe del impacto que genera desde el punto de vista ecoeconómico, clínico y la morbilidad asociada al tratamiento quirúrgico y por ende, de las complicaciones que eso conlleva, a pesar de ser una entidad de frecuente consulta y asociada a ingresos por urgencias, infecciones del tracto urinario, afectación de la función renal y realización de múltiples procedimientos quirúrgicos. ${ }^{1,2}$

La incidencia de urolitiasis varía considerablemente, siendo la tercera causa de consulta urológica en el mundo. ${ }^{3} \mathrm{Su}$ prevalencia varía dependiendo de factores geográficos como el clima, la etnia, la dieta y factores genéticos. ${ }^{1,3}$ No existen datos precisos, pero en general, el riesgo de padecer urolitiasis es mayor en países con estándares de vida altos ${ }^{2}$ (5\% al $9 \%$ en Europa, $12 \%$ en Canada, $13 \%$ al 15\% en Estados Unidos) y es notablemente alta en Emiratos Árabes Unidos y Arabia Saudita con una prevalencia aproximada del $20 \%{ }^{4}$ Se ha reportado en algunos estudios un incremento superior al $37 \%$ de la incidencia de enfermedad litiasica, atribuido a los cambios en el clima. ${ }^{1,5}$

En Sudamérica no se encuentran datos claros disponibles sobre la incidencia y prevalencia de la enfermedad. En Colombia no se encuentran datos sobre la prevalencia de la enfermedad litiasica en el Instituto Nacional de Salud; y las entidades administradoras de los recursos en salud, no tienen información pública sobre los costos asociados al tratamiento.
La urolitiasis tiene alto riesgo de recurrencia, casi del 50\% en personas que consultan al menos una vez por episodio de cólico renal. Ese riesgo se determina principalmente por la causa de la formación del cálculo (metabólica o anatómica). ${ }^{1,3}$

El tratamiento quirúrgico de la urolitiasis del tracto urinario superior, ha evolucionado de forma ostensible desde la realización de la primera nefrolitotomía percutánea descrita por Ferstran y Johanssen en $1976 .^{6}$

En general, la nefrolitotomía percutánea es un procedimiento seguro con una tasa específica de complicaciones baja. Sin embargo, las complicaciones globales pueden llegar a ser de un $83 \%$ incluyendo urinomas, transfusiones y fiebre. Las complicaciones mayores como sepsis, lesión pleural, lesión del colon son raras (del 3\% al $15 \%)$. Las comorbilidades como falla renal, diabetes mellitus, obesidad mórbida y enfermedad pulmonar, aumentan ese riesgo. $^{7-12}$

Existe poca información en Colombia sobre la evaluación de las características de los pacientes con diagnóstico de urolitiasis y especialmente sobre la aparición de complicaciones cuando son sometidos a procedimientos quirúrgicos de alta complejidad como es la nefrolitotomía percutánea. ${ }^{13}$ En Colombia, ese riesgo no está medido en los diferentes estudios.

En Medellín existe una escasa publicación al respecto de complicaciones quirúrgicas específicas con la nefrolitotomía percutánea. ${ }^{14}$ Incluso no hay información sobre su prevalencia. Esa falta de información genera la imposibilidad de prever o desarrollar estrategias de control para mitigación 
del riesgo de aparición, y así disminuir la morbilidad asociada a los procedimientos y sus costos para el sistema de salud.

Por lo anterior, cada vez se hace más importante conocer y medir los riesgos que se asumen al realizar tratamientos de alta complejidad en masas litiasicas mayores sobre todo teniendo en cuenta un problema de tanto peso como es la urolitiasis. Por ello el propósito de este artículo, fue determinar la prevalencia de las complicaciones en nefrolitotomía percutánea sobre algunas variables demograficas, clínicas e imaginológicas.

\section{Métodos}

Se realizó un estudio observacional, descriptivo y retrospectivo. Se describieron y explicaron características demográficas, clínicas e imaginológicas y se analizaron las variables de los pacientes con urolitiasis compleja sometidos a nefrolitotomía percutánea. La finalidad del estudio fue determinar la prevalencia de las complicaciones mayores durante nefrolitotomía percutánea en la población de una clínica de tercer nivel de la ciudad de Medellín - Colombia durante los años 2015 a 2017.

Se utilizó la historia clínica como fuente secundaria de información. Los criterios de inclusión fueron los registros de pacientes con 18 años o más con diagnóstico de urolitiasis del tracto urinario superior confirmado por tomografía y que fueron llevados a cirugía percutánea entre 2015 y 2017 en una clínica de tercer nivel de Medellín. Además, se incluyeron los registros completos de la historia clínica prequirúrgica y hasta el primer mes posterior a la cirugía.

Los criterios de exclusión fueron cirugía fallida definida como imposibilidad para generar un trayecto percutáneo al tracto urinario superior, ausencia de descripción de urocultivo prequirúrgico y tratamiento cuando su resultado era positivo en la historia clínica.

Para el análisis de la información, se utilizaron los programas SPSS versión 21, Epidat 3.1 y para la elaboración de gráficos e informe final, herramientas de Office.

\section{Resultados}

En total se analizaron 176 registros de pacientes a los que se les realizó nefrolitotomía percutánea. De ellos, el 60,2\% correspondía a mujeres. En cuanto a la edad, presentó una distribución normal, con una media de 45,1 años ( $\mathrm{DE} \pm 12,5$ años). La mayoría tenían entre 32,6 y 57,6 años.

El 26,1\% de los pacientes no tenían patologías previas, el $22,7 \%$ eran hipertensos, $8,5 \%$ diabéticos y el $42,6 \%$ presentaban otras patologías. Del total de pacientes, el $18,8 \%$ habían recibido manejo quirúrgico previo para el mismo cálculo. El 6,3\% presentaban nefrostotomía previa a la cirugía y $12,5 \%$ cateter doble J.

Al revisar los urocultivos, el 22,2\% del total de los pacientes tenían bacteriuria prequirúrgica y de ellos el patógeno más frecuente fue la Echerichia Coli 51,3\% (- Tabla 1).

El 15,9\% de los pacientes tenían cálculos coraliformes completos, $37,5 \%$ incompletos y el 37,5\% de los pacientes tenían sincrónicamente cálculos en ambos riñones. De todos los pacientes el 30\% de los cálculos se encontraban en la
Tabla 1 Características clínicas

\begin{tabular}{|l|l|l|l|}
\hline Categoría & Variable & $\boldsymbol{n}$ & $\%$ \\
\hline Antecedente patologico & Ninguno & 46 & 26,1 \\
\cline { 2 - 4 } & $\mathrm{HTA}$ & 40 & 22,7 \\
\cline { 2 - 4 } & $\mathrm{DM}$ & 15 & 8,5 \\
\cline { 2 - 4 } & Otros & 75 & 42,6 \\
\hline \multirow{4}{*}{$\begin{array}{l}\text { Cirugía previa para el } \\
\text { mismo cálculo }\end{array}$} & No & 143 & 93,8 \\
\cline { 2 - 4 } & $\mathrm{Si}$ & 33 & 6,3 \\
\hline Nefrostomía previa & No & 165 & 93,8 \\
\cline { 2 - 4 } & $\mathrm{Si}$ & 11 & 6,3 \\
\hline Doble J previo & No & 154 & 87,5 \\
\cline { 2 - 4 } & Si & 22 & 12,5 \\
\hline Urocultivo prequirúrgico & Negativo & 137 & 77,8 \\
\cline { 2 - 4 } & Positivo & 39 & 22,2 \\
\hline Bacteriuria prequirúrgica & Ninguno & 137 & 77,8 \\
\cline { 2 - 4 } & E Coli & 20 & 11,4 \\
\cline { 2 - 4 } & P Mirabilis & 7 & 4,0 \\
\cline { 2 - 4 } & Otro & 12 & 6,8 \\
\hline
\end{tabular}

pelvis renal, $35,8 \%$ en el grupo calicial inferior y $26,1 \%$ en el superior (-Tabla 2 ).

El total de los pacientes analizados fueron manejados con técnica en prono para la realización de la cirugía. El 81,8\% de los pacientes requirió una punción para alcanzar trayecto efectivo, de los cuales el $42,6 \%$ fue en el caliz superior y el $40,3 \%$ en el inferior. El 7,4\% de los pacientes requirieron punción intercostal. El 58,5\% de los pacientes quedó libre de cálculos durante la cirugía.

El $54,0 \%$ de los pacientes quedaron derivados simultáneamente con doble J y nefrostomía al final del procedimiento, el 39,2\% requirió unicamente nefrostomía, el $4,5 \%$ con doble J y el $2,3 \%$ de los pacientes no requirieron ningún método de derivación urinaria. Al 3,4\% de los pacientes se les realizó bloqueo paravertebral como mecanismo analgésico realizado por urólogo al principio de la cirugía. La mediana de hospitalización fue de 2,5 días $(\mathrm{RIQ}=2)$ con una distribución no normal. Todos los pacientes con complicaciones mayores tuvieron una hospitalización de 3 o más días (-Tabla 3 )

La prevalencia de complicaciones mayores, definidas por la clasificación Clavien Dindo IIIA en adelante, fue del 11,4\%. El $83 \%$ de los pacientes no presentaron ningún tipo de complicación (-Tabla 4 ).

Ninguno de los pacientes requirió transfusión intraoperatoria, el 5,6\% requirió al menos dos unidades de GRE postquirúrgicas. Dos pacientes presentaron complicaciones vasculares: un caso presentó un hematoma perirenal anemizante postoperatorio y otro paciente presentó hematuria anemizante secundaria a sangrado activo arterial al tracto urinario, las cuales se manejaron con embolización selectiva por el grupo de hemodinamia de la clínica con buena respuesta. 
Tabla 2 Características imaginológicas

\begin{tabular}{|c|c|c|c|}
\hline Variable & Categoría & $n$ & $\%$ \\
\hline \multirow[t]{2}{*}{ Lateralidad } & Izquierdo & 80 & 45,5 \\
\hline & Derecho & 96 & 54,5 \\
\hline \multirow[t]{2}{*}{ Bilateral } & No & 110 & 62,5 \\
\hline & Sí & 66 & 37,5 \\
\hline \multirow{3}{*}{$\begin{array}{l}\text { Cálculo } \\
\text { coraliforme }\end{array}$} & No & 82 & 46,6 \\
\hline & Coraliforme incompleto & 66 & 37,5 \\
\hline & Coraliforme completo & 28 & 15,9 \\
\hline \multirow{10}{*}{$\begin{array}{l}\text { Localización } \\
\text { del cálculo }\end{array}$} & Sin información & 3 & 1,7 \\
\hline & Cáliz superior & 14,0 & 8,0 \\
\hline & Cáliz medio & 10 & 5,7 \\
\hline & Cáliz inferior & 29 & 16,5 \\
\hline & Cáliz superior y medio & 7 & 4 \\
\hline & $\begin{array}{l}\text { Cáliz superior medio } \\
\text { e inferior }\end{array}$ & 25 & 14,2 \\
\hline & Cáliz superior e inferior & 8 & 4,5 \\
\hline & Cáliz medio e inferior & 26 & 14,8 \\
\hline & Pelvis renal & 53 & 30,1 \\
\hline & Ureter & 1 & 0,6 \\
\hline
\end{tabular}

Tabla 3 Características quirúrgicas

\begin{tabular}{|c|c|c|c|}
\hline Variable & Categoría & $n$ & $\%$ \\
\hline \multirow{3}{*}{$\begin{array}{l}\text { Número de } \\
\text { punciones }\end{array}$} & 1 & 148 & 84,1 \\
\hline & 2 & 26 & 14,8 \\
\hline & 3 & 2 & 1,1 \\
\hline \multirow{3}{*}{$\begin{array}{l}\text { Sitio de la } \\
\text { primera punción } \\
\text { (grupo calicial) }\end{array}$} & Superior & 75 & 42,6 \\
\hline & Medio & 30 & 17 \\
\hline & Inferior & 71 & 40,3 \\
\hline \multirow{4}{*}{$\begin{array}{l}\text { Sitio de la } \\
\text { segunda punción } \\
\text { (grupo calicial) }\end{array}$} & No requirió & 148 & 84,1 \\
\hline & Cáliz superior & 12 & 6,8 \\
\hline & Cáliz medio & 8 & 4,5 \\
\hline & Cáliz inferior & 8 & 4,5 \\
\hline \multirow{4}{*}{$\begin{array}{l}\text { Punción } \\
\text { intercostal }\end{array}$} & No & 136 & 77,3 \\
\hline & Intercostal 9-10 & 2 & 1,1 \\
\hline & Intercostal 11-12 & 11 & 6,3 \\
\hline & Sin información & 27 & 15,3 \\
\hline \multirow{5}{*}{$\begin{array}{l}\text { Método de } \\
\text { fragmentación }\end{array}$} & Ninguno & 4 & 2,3 \\
\hline & Litotriptor neumático & 157 & 89,2 \\
\hline & Litotriptor ultrasónico & 5 & 2,8 \\
\hline & Laser de holmio & 7 & 4,0 \\
\hline & $\begin{array}{l}\text { Litotriptor neumático } \\
\text { y ultrasónico }\end{array}$ & 3 & 1,7 \\
\hline
\end{tabular}

La complicación pulmonar y la infecciosa fueron las más frecuentes siendo cada una del 6,3\%. Un 6,8\% de los pacientes requirió algún tipo de intervención por cálculos residuales durante los primeros 30 días del postquirúrgico. No se presentó ninguna complicación intestinal.
Tabla 4 Complicaciones

\begin{tabular}{|l|l|l|}
\hline Tipo de complicación & $\boldsymbol{n}$ & $\%$ \\
\hline Complicaciones mayores (Clavien Dindo IIIA-V) & 20 & 11,4 \\
\hline Complicación Pulmonar & 11 & 6,3 \\
\hline Complicación intestinal & 0 & 0,0 \\
\hline Complicación infecciosa & 11 & 6,3 \\
\hline Complicación tipo colección & 3 & 1,7 \\
\hline Complicación por cálculos residuales & 12 & 6,8 \\
\hline Complicación vascular & 2 & 1,1 \\
\hline Complicación tipo fístula & 3 & 1,7 \\
\hline
\end{tabular}

Tabla 5 Tratamiento de Complicaciones

\begin{tabular}{|c|c|c|c|}
\hline Variable & Categoría & $n$ & $\%$ \\
\hline \multirow{5}{*}{$\begin{array}{l}\text { Manejo de } \\
\text { complicación } \\
\text { pulmonar }\end{array}$} & Ninguna & 166 & 94,3 \\
\hline & Drenaje percutáneo & 1 & 0,6 \\
\hline & Toracostomía cerrada & 3 & 1,7 \\
\hline & Toracoscopia & 4 & 2,3 \\
\hline & Terapia respiratoria & 2 & 1,1 \\
\hline \multirow{3}{*}{$\begin{array}{l}\text { Manejo de } \\
\text { Complicación } \\
\text { intestinal }\end{array}$} & Ninguna & 0 & 0 \\
\hline & Drenaje percutáneo & 0 & 0 \\
\hline & Derivación Intestinal & 0 & 0 \\
\hline \multirow{2}{*}{$\begin{array}{l}\text { Manejo de } \\
\text { Complicación } \\
\text { infecciosa }\end{array}$} & Ninguno & 165 & 93,8 \\
\hline & Antibiótico & 11 & 6,3 \\
\hline \multirow{3}{*}{$\begin{array}{l}\text { Manejo de } \\
\text { Complicación tipo } \\
\text { colección }\end{array}$} & Ninguna & 174 & 98,9 \\
\hline & Drenaje percutáneo & 1 & 0,6 \\
\hline & Drenaje abierto & 1 & 0,6 \\
\hline \multirow{3}{*}{$\begin{array}{l}\text { Manejo de } \\
\text { Complicación por } \\
\text { cálculos residuales }\end{array}$} & Ninguno & 165 & 93,8 \\
\hline & Derivación & 1 & 0,6 \\
\hline & $C x$ & 10 & 5,7 \\
\hline \multirow{3}{*}{$\begin{array}{l}\text { Manejo de } \\
\text { Complicación } \\
\text { vascular }\end{array}$} & Ninguna & 174 & 98,9 \\
\hline & $\begin{array}{l}\text { Embolización } \\
\text { (Rx intervencionista) }\end{array}$ & 2 & 1,1 \\
\hline & Nefrectomía & 0 & 0 \\
\hline \multirow{3}{*}{$\begin{array}{l}\text { Manejo de } \\
\text { Complicación tipo } \\
\text { fístula nefrocutánea }\end{array}$} & Ninguna & 173 & 98,3 \\
\hline & Nefrostomía & 2 & 1,1 \\
\hline & Doble J & 1 & 0,6 \\
\hline
\end{tabular}

De los 11 pacientes con complicaciones pulmonares, el $36 \%$ requirió manejo con toracoscopia y el $27,3 \%$ toracostomía cerrada. El 18,2\% requirió manejo con terapia respiratoria.

De los 11 pacientes con complicaciones infecciosas el tratamiento dado fue antibiótico en el $100 \%$ de las veces. Las colecciones se presentaron en dos pacientes, uno se manejó con drenaje percutáneo y otro con drenaje abierto.

El 1,7\% de los pacientes presentaron fístulas urinarias y 1,1\% fueron manejados con nefrostomía percutánea (-Tabla 5). 


\section{Discusión}

La enfermedad litiásica del tracto urinario es un motivo de consulta común en la práctica médica. El espectro de sintomatología puede ser muy amplio, desde un hallazgo incidental imaginológico sin ser causante de dolor, hasta alteración de la función renal con procesos infecciosos sobreagregados que pueden llegar a comprometer la vida. ${ }^{15}$

El manejo de una patología de alta incidencia y poco prevenible como la urolitiasis y la potencial morbilidad inducida por su tratamiento, genera un gran impacto en cualquier sistema de salud. ${ }^{16}$

La prevalencia en el mundo de la enfermedad litiásica urinaria varía de $1 \%$ al 20\%. ${ }^{4}$ Hacia 1965 había una incidencia mundial de litiasis de 54,2 por 100.000 habitantes, mientras que en 2005 se encontró 114,3 por cada 100.000 habitantes. ${ }^{17}$ Se ha evidenciado que tiende a ser más frecuente en pacientes masculinos, en comparación con el sexo femenino, teniendo una tasa de $1,49: 1 .^{18}$ Es raro encontrar urolitiasis antes de los 20 años, por lo general en hombres la edad pico varía entre los 20-70 años, siendo más común en la tercera y cuarta década de vida, mientras que en mujeres, la distribución es bimodal, teniendo el primer pico entre los 30-39 años, y el segundo pico 60-69 años, por lo que se han generado hipótesis sobre el papel protector estrogénico. ${ }^{20,21}$ Sin embargo, el presente estudio se encontró que el $60 \%$ de los pacientes eran mujeres.

El actual estándar de oro imaginológico de diagnóstico es la tomografía sin contraste, un examen cada vez más disponible en la práctica médica, incluso en países en vía de desarrollo. Teniendo una sensibilidad del 96,6\% y una especificidad del 94,9\%. ${ }^{21}$ Es importante al interpretar las imágenes conocer el tamaño, la localización, la densidad y la distancia piel-cálculo para enfocar el manejo, además de otros hallazgos como dilatación de sistemas colectores y cambios sugestivos de inflamación y proceso infeccioso sobreagregado. En la presente investigación se encontró que la mayoría de los cálculos estaban en la pelvis renal $(30,0 \%)$ y eran coraliformes hasta en el 53,4\% de los casos. El 37,5\% de los casos analizados presentaron enfermedad sincrónica bilateral. Esos datos son indicadores de la alta correlación imaginológica con la indicación de nefrolitotomía percutánea, además que la mediana de tamaño de los cálculos fue de $25 \mathrm{~mm}$. La guía europea de urología sobre el manejo de los cálculos renales, ${ }^{22}$ recomienda que en cálculos renales mayores a $20 \mathrm{mms}$ la primera línea de tratamiento es la nefrolitotomía percutánea, y como segunda opción cirugía retrógrada o litotripsia extracorpórea. Igualmente aclaran que para cálculos pequeños $(<10 \mathrm{mms})$, lo ideal sería realizar litotripsia extracorpórea, siempre y cuando no cumplan factores que no favorezcan su uso como cálculos resistentes a ondas de choque (oxalato de calcio monohidratado, brushita o cistina), ángulo pielo-infundibular angosto, cáliz inferior largo $>10 \mathrm{mms}$, o un infundíbulo estrecho ( $<5 \mathrm{mms})^{23}$

La nefrolitotomía percutánea es una técnica que tiene sus inicios en la década de 1970 descrita por Ferstran y Johanssen. ${ }^{6,7}$ Aunque desde sus inicios no fue ampliamente usada por la presencia de la litotripsia extracorpórea, en las siguientes décadas empezó a aumentar su uso, hasta llegar a ser el estándar de oro hoy en día, principalmente para cálculos intrarrenales mayores a $20 \mathrm{mms}$.

Aunque no es muy clara en la literatura la cantidad de procedimientos requeridos para obtener una adecuada curva de aprendizaje para la realización de nefrolitotomía percutánea, Schilling y colaboradores en el 2011, en un estudio retrospectivo sugirieron que se requerían por lo menos 35 procedimientos, evidenciándose un menos tiempo quirúrgico y uso de fluoroscopio, además de menor tasa de complicaciones y retratamientos. ${ }^{24}$

Si bien la nefrolitotomía percutánea es un procedimiento mínimamente invasivo, no está libre de dolor posoperatorio, una de las medidas tomadas en el intraoperatorio es un bloqueo paravertebral ipsilateral, ${ }^{25}$ inicialmente una técnica usada en cirugía de tórax ${ }^{26}$ y cirugías para cáncer de mama. ${ }^{27}$ En los últimos años extendido al uso en endourología. ${ }^{28,29}$ En esta investigación, se evidencia que apenas se inicia la implementación de la técnica pero no se vieron mayores complicaciones en ese grupo de pacientes. Sin embargo, la muestra fue pequeña para tomar una conclusión definitiva y no fue medido el dolor postquirúrgico.

En el presente estudio las complicaciones mayores presentaron una prevalencia del $11,4 \%$, de las cuales el $75 \%$ eran del sexo femenino. La media de la edad fueron 44,95 años con una distribución normal y una desviación estandar de $\pm 12,5$ años. El $55 \%$ de los pacientes no presentaban patologías previas. El 5\% de los pacientes con complicaciones mayores habían tenido cirugías previas para el mismo cálculo y el 5\% de los pacientes no presentaban nefrostomía y ninguno doble J. El 20\% de los pacientes presentaban urocultivo positivo siendo por E Coli en el 15\%, los cuales recibieron terapia antibiótica dirigida previa a la cirugía. El 60\% de los cálculos eran coraliformes, y se encontraban en el caliz inferior en el $45 \%$, siendo bilaterles en el $40 \%$ de los casos. El $90 \%$ de los pacientes no requirió punción intercostal.

Las complicaciones de carácter infeccioso pueden presentarse después de cualquier procedimiento quirúrgico. Además de todas las medidas de asepsia tomadas en cirugía, debe tenerse en cuenta que es una cirugía del tracto urinario, y en lo posible contar con un urocultivo negativo previo al acto quirúrgico. ${ }^{30}$ En el presente estudio 22,2\% de los casos de complicación presentaban urocultivo positivo, aislando $E$ Coli en el 11,4\%. Las complicaciones infecciosas fueron del $6,3 \%$ de los datos analizados y el enfoque de tratamiento fue guiado según el aislamiento microbiológico con terapia antibiótica requiriendo drenaje percutáneo en el $0,6 \%$ y abierto el $0,6 \%$.

En algunas series, las hemorragias pueden llegar a tener una incidencia de hasta un 34\% ${ }^{30,32,42,44}$ generalmente asociado a la presencia de un parénquima grueso y un mayor tiempo operatorio. Aunque no se describió el dato de sangrado intraoperatorio, las transfusiones son un buen estimador de ese evento y no superó el 5,6\%. Para disminuir el riesgo de sangrado se recomienda además de una punción limpia y una dilatación secuencial, tener un adecuado manejo de las pinzas durante la extracción de fragmentos 
de cálculos, y reposicionar la camisa en caso de sangrados. Contar con un grupo de hemodinamia en la instituición donde se realice el procedimiento es casi un imperativo, pues la mayoría de los casos según la literatura son controlados con embolización selectiva según los hallazgos en arteriografía como fue el caso de los dos pacientes presentados en el estudio.

En pocos reportes de la literatura se menciona la tasa de fragmentación incompleta y migración distal de cálculos, que dependiendo del tamaño, se requerirá de un segundo tiempo quirúrgico, en la mayoría de los casos, retrógrado para la extracción de cálculos residuales distales. Según los hallazgos intraoperatorios se debe decidir dejar cateter JJ para evitar cólicos nefríticos en el posoperatorio temprano. El 6,8\% de los pacientes del estudio presentó complicaciones por cálculos residuales que requirieron intervención, la cual requirió cateter doble J en el 0,6\% de los pacientes y en el 5,7\% restante manejo endoscópico.

Las complicaciones torácicas según la serie de casos puede llegar a tener una incidencia hasta del $15,3 \%$, con una gama muy amplia de entidades: neumotórax, hidrotórax, hemotórax, urinotórax, fístulas nefro-pleurales $(0,87 \%)$ y combinación de varias de éstas. En el presente estudio se encontraron 6,3\% de complicaciones pulmonares, asociado a punciones supracostales. Al tener un volumen de líquido mayor a $250 \mathrm{cc}$, debe evaluarse la opción de una sonda a tórax(33). La mayoría de los pacientes del estudio requirieron manejo conservador, pero el 2,3\% de los casos requirió cirugía con toracoscopia para completar el tratamiento. Esas complicaciones menos frecuentes son el hemotórax coagulado y ruptura del árbol respiratorio que puede conllevar a la formación de fístulas broncopleurales. Tener en cuenta durante la punción el momento óptimo para realizarla es en espiración sostenida, por lo cual es importante un grupo de anestesia familiarizado con el procedimiento.

Las lesiones intestinales son más comunes en posiciones prono por la disposición anatómica intestinal, sin embargo en punciones en supino puede ocurrir, por lo que siempre es importante tener una imagen prequirúrgica que descarte un colon retrorrenal. ${ }^{34}$ En el presente estudio no se presentó ningún evento intestinal asociado a la nefrolitotomía percutánea. En caso de evidenciarse una perforación intestinal durante la cirugía, debe retirarse la camisa de cavidades renales y posicionarse la sonda de nefrostomía en lumen intestinal, teniendo dos objetivos: el primero es distanciar el asa intestinal del riñón y disminuir la presencia de futuras fístulas reno-entéricas, y el segundo es garantizar el cierre del defecto de la pared intestinal. A los ocho días debe realizarse evaluación imaginológica de tránsito intestinal contrastada y en caso de no haber extravasación puede retirarse la sonda de nefrostomía. Otra medida importante para tener en cuenta es la colocación de cateter ureteral JJ.

Otras complicaciones de menor complejidad y frecuencia que deberían ser tenidas en cuenta (sobrecarga hídrica, hipotermia, lesiones por posición en especial del plexo braquial al posicionar el paciente en prono, embolismo aéreo, perforación y extravasación, fístulas nefrocutáneas y siembras tumorales ${ }^{35}$ ), no fueron encontradas en esta investigación.

\section{Conclusiones}

La prevalencia de complicaciones mayores que se encontró en el estudio fue del $11,4 \%$ siendo el $75 \%$ mujeres. La media de la edad fueron 44,95 años ( $D E \pm 12,5$ años) con $55 \%$ de los pacientes sin antecedentes patológicos. El $5 \%$ de los pacientes con complicaciones mayores habían tenido cirugías previas para el mismo cálculo y el $5 \%$ de los pacientes no presentaban nefrostomía y ninguno doble J. El 20\% de los pacientes presentaban urocultivo positivo siendo por E Coli en el 15\%. El $60 \%$ de los cálculos eran coraliformes, y se encontraban en el caliz inferior en el 45\%, siendo bilaterales en el $40 \%$ de los casos. El $90 \%$ de los pacientes no requirió punción intercostal. Esa prevalencia está a tono con la estadística actual encontrada en la literatura. Las complicaciones que más se encontraron fueron por cálculos residuales en el 6,8\%, pulmonar en el 6,3\% e infecciosa en el 6,3\%. El tratamiento utilizado para el manejo en el 6,3\% fue antibioticoterapia, seguido de cirugía por cálculos residuales y toracoscopia en el 2,3\%. El 1,1\% de los pacientes requirió el manejo con embolización por radiología intervencionista.

\section{Responsabilidades éticas}

Protección de personas y animales. Los autores declaran que los procedimientos seguidos estuvieron de acuerdo con las normas éticas del comité de experimentación humana responsable y también con la Asociación Médica Mundial y la Declaración de Helsinki.

Confidencialidad de los datos. Los autores declaran que han seguido los protocolos de su centro de trabajo sobre la publicación de datos de pacientes.

Derecho a la privacidad y consentimiento informado. Los autores han obtenido el consentimiento informado de los pacientes y/o sujetos referidos en el artículo. Este documento obra en poder del autor de correspondencia.

Conflicto de intereses

Los autores declaran no tener ningún conflicto de intereses.

\section{Referencias}

1 Stamatelou KK, Francis ME, Jones CA, Nyberg LM, Curhan GC. Time trends in reported prevalence of kidney stones in the United States: 1976-1994. Kidney Int 2003;63(05):1817-1823

2 Fisang C, Anding R, Müller SC, Latz S, Laube N. Urolithiasis-an interdisciplinary diagnostic, therapeutic and secondary preventive challenge. Dtsch Arztebl Int 2015;112(06):83-91

3 Alatab S, Pourmand G, El Howairis MelF, et al. National Profiles of Urinary Calculi: a Comparison Between Developing and Developed Worlds. Iran J Kidney Dis 2016;10(02):51-61

4 Romero V, Akpinar H, Assimos DG. Kidney stones: a global picture of prevalence, incidence, and associated risk factors. Rev Urol 2010;12(2-3):e86-e96

5 Borg M, Bi P, Nitschke M, Williams S, McDonald S. The impact of daily temperature on renal disease incidence: an ecological study. Environ Health 2017;16(01):114 
6 Fernström I, Johansson B. Percutaneous pyelolithotomy. A new extraction technique. Scand J Urol Nephrol 1976;10(03):257-259

7 Michel MS, Trojan L, Rassweiler JJ. Complications in percutaneous nephrolithotomy. Eur Urol 2007;51(04):899-906, discussion 906

8 Taylor E, Miller J, Chi T, Stoller ML. Complications associated with percutaneous nephrolithotomy. Transl Androl Urol 2012;1(04): 223-228

9 Watterson JD, Soon S, Jana K. Access related complications during percutaneous nephrolithotomy: urology versus radiology at a single academic institution. J Urol 2006;176(01):142-145

10 Ün S, Çakır V, Köse O, Türk H, Yılmaz Y. Colon perforation during percutaneous nephrolithotomy and fistula closure with Spongostan following conservative therapy. Can Urol Assoc J 2015;9(5-6):E313-E315

11 Tefekli A, Ali Karadag M, Tepeler K, et al. Classification of percutaneous nephrolithotomy complications using the modified clavien grading system: looking for a standard. Eur Urol 2008;53(01):184-190

12 Dindo D, Demartines N, Clavien P-A. Classification of surgical complications: a new proposal with evaluation in a cohort of 6336 patients and results of a survey. Ann Surg 2004;240(02):205-213

13 Villarraga JDA. Nefrolitotomía percutánea vs. tubeless.? Es realmente necesaria la derivación urinaria? Rev Urol Colomb. 2016;25(03):229-230

14 Cabrales Hessen M, Céspedes Orrego CA. Nefrolitotomía percutánea en posición supina: reporte de una serie de casos. Rev Urol Colomb 2012;21(01):

15 Türk C, Petřík A, Sarica K, et al. EAU guidelines on interventional treatment for urolithiasis. Eur Urol 2016;69(03):475-482

16 Chi T, Taylor E, Stoller ML. The days of cost effective management for nephrolithiasis are already upon us. Transl Androl Urol 2014;3 (03):284

17 Yasui T, Iguchi M, Suzuki S, Kohri K. Prevalence and epidemiological characteristics of urolithiasis in Japan: national trends between 1965 and 2005. Urology 2008;71(02):209-213

18 Scales CD Jr, Smith AC, Hanley JM, Saigal CS; Urologic Diseases in America Project. Prevalence of kidney stones in the United States. Eur Urol 2012;62(01):160-165

19 Johnson CM, Wilson DM, O'Fallon WM, Malek RS, Kurland LT. Renal stone epidemiology: a 25-year study in Rochester, Minnesota. Kidney Int 1979;16(05):624-631

20 Niemann T, Kollmann T, Bongartz G. Diagnostic performance of low-dose CT for the detection of urolithiasis: a meta-analysis. AJR Am J Roentgenol 2008;191(02):396-401

21 Professionals S-O. Urolithiasis [Internet]. Uroweb. 2015 [citado el 17 de junio de 2017]. Disponible en: http://uroweb.org/guideline/ urolithiasis./
22 Manikandan R, Gall Z, Gunendran T, Neilson D, Adeyoju A. Do anatomic factors pose a significant risk in the formation of lower pole stones? Urology 2007;69(04):620-624

23 Schilling D, Gakis G, Walcher U, Stenzl A, Nagele U. The learning curve in minimally invasive percutaneous nephrolitholapaxy: a 1-year retrospective evaluation of a novice and an expert. World $\mathrm{J}$ Urol 2011;29(06):749-753

24 Cowie B, McGlade D, Ivanusic J, Barrington MJ. Ultrasound-guided thoracic paravertebral blockade: a cadaveric study. Anesth Analg 2010;110(06):1735-1739

25 Vogt A, Stieger DS, Theurillat C, Curatolo M. Single-injection thoracic paravertebral block for postoperative pain treatment after thoracoscopic surgery. $\mathrm{Br} J$ Anaesth 2005;95(06): 816-821

26 Schnabel A, Reichl SU, Kranke P, Pogatzki-Zahn EM, Zahn PK. Efficacy and safety of paravertebral blocks in breast surgery: a meta-analysis of randomized controlled trials. Br J Anaesth 2010; 105(06):842-852

27 Rashwan MD, Elbealy ME. A comparison of the effects of epidural anesthesia, lumbar paravertebral block and general anesthesia in percutaneous nephrolithotomy. J Med Sci. 2008;8(02):170-176

28 Ak K, Gursoy S, Duger C, et al. Thoracic paravertebral block for postoperative pain management in percutaneous nephrolithotomy patients: a randomized controlled clinical trial. Med Princ Pract 2013;22(03):229-233

29 Dehong C, Liangren L, Huawei L, Qiang W. A comparison among four tract dilation methods of percutaneous nephrolithotomy: a systematic review and meta-analysis. Urolithiasis 2013;41(06): 523-530

30 Horan TC, Andrus M, Dudeck MA. CDC/NHSN surveillance definition of health care-associated infection and criteria for specific types of infections in the acute care setting. Am J Infect Control 2008;36(05):309-332

31 Gremmo E, Ballanger P, Doré B, Aubert J. [Hemorrhagic complications during percutaneous nephrolithotomy. Retrospective studies of 772 cases]. Prog Urol 1999;9(03):460-463

32 Lallas CD, Delvecchio FC, Evans BR, Silverstein AD, Preminger GM, Auge BK. Management of nephropleural fistula after supracostal percutaneous nephrolithotomy. Urology 2004;64 (02):241-245

33 Noor Buchholz NP. Colon perforation after percutaneous nephrolithotomy revisited. Urol Int 2004;72(01):88-90

34 Percutaneous Nephrolithotomy Complications in 671 Consecutive Patients: A Single-Center Experience - ProQuest [Internet]. [citado el 11 de septiembre de 2017]. Disponible en: https://search. proquest.com/openview/0f6f6f6ffad2ff154a1664e76065f041/1? pq-origsite $=$ gscholar $\& \mathrm{cbl}=105751$ 